\title{
L'instabilité des prix agricoles : réflexion sur les causes et les implications de la flambée des prix
}

\author{
Françoise GÉRARD ${ }^{1}$ \\ Marie Gabrielle PIKETTY² \\ Jean-Marc BOUSSARD ${ }^{3}$ \\ ${ }^{1}$ CIRAD, UMR MOISA \\ $<$ francoise.gerard@cirad.fr> \\ ${ }^{2}$ CIRAD, UMR MOISA et Université de Sao Paolo \\ ${ }^{3}$ Académie d'Agriculture
}

\begin{abstract}
This article analyzes the recent surge in market prices of raw materials, as the result of a feverish episode, characteristic of these markets when they are not regulated. Are these fluctuations exogenous or endogenous? Significant for understanding the system or not critical? Most models assume that they are negligible and requirements arising from this are those of a minimal state intervention in economic life and "faith in free markets" as policy development. However, we must acknowledge the limited progress achieved in recent decades, in the situation of low-income countries, particularly in Africa. The requirements from the dominant models in economics have been ineffective, leading to reflect on their ability to describe the process and to focus on opportunities for improvement. The model ID3 CIRAD is an attempt in that direction.
\end{abstract}

Key words: price fluctuation, dominant models in economics, model ID3 CIRAD
L'instabilité des prix est une caractéristique majeure des marchés de produits agricoles. On pourrait à peu près dire la même chose des marchés financiers. Le phénomène a suscité bien des travaux d'économistes, à partir de deux idées complètement différentes, conduisant à des politiques opposées. Dans la première interprétation, l'instabilité est exclusivement le résultat de chocs exogènes - aléas climatiques, choc macroéconomique lié à une mauvaise gouvernance, etc. Dans la seconde, le fonctionnement même des marchés est susceptible d'entraîner des fluctuations qui s'ajoutent aux chocs extérieurs au système et qui peuvent apparaître même en l'absence de tout choc extérieur.

Cette différence d'interprétation a des conséquences importantes pour la représentation des phénomènes économiques et surtout pour les prescriptions qui en découlent en matière de politique économique. L'idée développée ici est que la récente flambée des prix des produits agricoles (2006-2008) est un «épisode fiévreux » $d$ 'origine essentiellement endogène, comme on en observe régulièrement sur les marchés libres de matières premières.

Dans une première section, les explications théoriques permettant de comprendre ce comportement des produits agricoles sont rappelées. On s'interroge ensuite sur l'intérêt de prendre en compte de tels phénomènes dans les modèles économiques, en analysant le point de vue retenu dans la plupart des modèles et les prescriptions qui en découlent en matière $d$ 'intervention sur les marchés (section 2). On rend compte enfin de travaux récents sur la question, menés au Cirad, où la prise en compte des caractéristiques particulières des marchés agricoles dans un modèle par ailleurs très classique, permet de représenter des fluctuations de prix similaires à celles observées (section 3). Ceci montre l'intérêt, au-delà des difficultés techniques, d'un renouvellement des modèles économiques afin de représenter la «dynamique complexe » des systèmes et attire l'attention sur un large champ de recherche encore peu exploré.

\section{Instabilité des prix et fonctionnement des marchés}

Dans la théorie néoclassique, les prix jouent un rôle essentiel, ils sont le vecteur de l'information, ils indiquent les raretés relatives des biens et permettent l'adaptation des comportements des producteurs et des consommateurs. Des décisions décentralisées peuvent ainsi mener à une allocation optimale des facteurs de production, si la «bonne concurrence » amène le prix à toujours se trouver égal au coût marginal de production. Dans ce contexte, l'intervention publique, en cassant la relation entre prix et coût marginal, est généralement source de distorsions et diminue le bien-être global. Il en est de même de l'instabilité, et pour les mêmes raisons : lorsque les prix varient trop vite, les coûts marginaux ne suivent pas, et on se trouve en permanence dans une situation "sous-optimale ». Mais d'où vient cette instabilité ?
Pour beaucoup d'économistes - en particulier les néoclassiques - l'instabilité des prix s'explique par des perturbations extérieures au système. Dans le cas des produits agricoles, la dépendance des rendements aux aléas climatiques, aux attaques de ravageurs et plus généralement à l'ensemble des conditions naturelles et sociales apporte une explication immédiate. Elle est le résultat de fluctuations aléatoires des volumes produits, aggravés par la rigidité de la demande propre aux produits alimentaires et qui implique de fortes variations des prix pour équilibrer le marché suite à de faibles variations des volumes commercialisés. Elle est alors représentée comme un aléa gaussien sur les fonctions d'offre. Mais est-on bien sûr de la pertinence de ce « modèle gaussien » ?

Mandelbrot [1, 2] distingue deux types d'aléas. Le hasard bénin, gaussien, disparaît lorsque l'échelle d'observation est suffisamment grossière. Au contraire, I'homothétie statique interne caractérise les «formes nouvelles de hasard ", ou aléa parétien (parce que la loi de probabilité correspondante est une « loi stable de Pareto »- loi de probabilité que Pareto avait à l'origine utilisée pour représenter la dispersion des revenus). La loi de Pareto possède des propriétés très différentes de celles de la loi de Gauss. En particulier, la disparition progressive de l'instabilité quand le nombre d'observations indépendantes augmente, qui est une caractéristique importante de la loi de Gauss, n'existe plus avec la loi de Pareto. Au contraire, les séries parétiennes sont caractérisées par l'invariance d'échelle : l'instabilité garde la même importance quelle que soit l'échelle d'observation. 
Pour expliquer ce phénomène, Mandelbrot [2] se réfère aux rôles des prévisions dans le mécanisme de formation des prix «la raison, à priori, est que l'offre et la demande, qui en principe doivent déterminer un prix, résultent toutes deux à la fois de facteurs objectifs et d'anticipations. Même si on accepte pour les premiers une approximation continue, les secondes peuvent changer du tout au tout à la suite d'un signal physique dont la durée et l'énergie sont négligeables, tel le proverbial " trait de plume ». Du coup la rationalité dans la détermination des prix risque fort de mener à des sauts, signifiant qu'elle est déstabilisante (p. 330)».

Ces mécanismes ont été depuis largement étudiés, en particulier sur les marchés financiers. Kindleberger [3] distingue ainsi les périodes de fonctionnement «normal » des marchés, des périodes de paniques, caractéristiques du fonctionnement des marchés financiers. II reprend la théorie des prévisions autoréalisatrices [4] parfaitement illustrée par les grandes séries de faillites bancaires, comme celle de 1929, où une crise de confiance amène les acteurs économiques à retirer simultanément leur dépôt bancaire et engendre l'impossibilité des banques à faire face à leurs créanciers... et ainsi la crise elle-même. Des comportements similaires s'observent sur les marchés des biens alimentaires. Lorsque les prix augmentent, les ménages qui en ont les moyens stockent, ce qui renforce la croissance des prix, confirmant les rumeurs de pénurie et amplifiant les comportements stockeurs. Dans la crise récente des produits alimentaires, la réaction des États, qui limitent les exportations pour protéger leurs populations, amplifient de la même façon la flambée des prix.

L'étude du fonctionnement des marchés de produits vivriers pendant les grandes crises, par exemple en Inde, lors de la famine de 1974 [5] ou en Indonésie lors de la crise financière $[6,26]$, met en évidence l'importance des anticipations, de la confiance des opérateurs ou au contraire des paniques, éventuellement déclenchées par des rumeurs infondées, dans les brusques flambées des prix des produits vivriers. On constate alors que la déviation réelle de la disponibilité de la marchandise, du moins à un niveau agrégé, n'est que de quelques pour-cent et qu'elle ne justifie en rien une telle augmentation des prix.

Ce sont les anticipations pessimistes qui expliquent le phénomène : la légère baisse de l'offre génère une faible augmentation des prix, mais du fait des prévisions des agents de la poursuite de la hausse (éventuellement fondée sur des informations plus ou moins vérifiées - la récolte est catastrophique, les entrepôts de l'état sont vides et ses difficultés financières empêchent le recours aux importations), les stocks privés augmentent et de moins en moins de vivres sont disponibles... les prix flambent... On se trouve dans la situation paradoxale où, comme le mentionne la $\mathrm{FAO}$ [7] à propos de I'Indonésie, les difficultés de fonctionnement des marchés mènent à une augmentation des prix qui, en générant des mouvements sociaux, accroissent le risque et font ainsi monter les prix, entraînant plus de difficultés pour le fonctionnement des marchés et générant ainsi un cercle vicieux à la hausse.

On voit bien ainsi la similitude entre marchés financiers et agricoles : le rôle central des anticipations, l'incertitude qui pèse sur celles-ci et leur volatilité. On peut alors, qu'ils s'agissent des marchés financiers ou de ceux de matières premières, opposer les périodes de fonctionnement normal, où les fluctuations traduisent les changements dans les raretés relatives et envoient aux agents les informations nécessaires à l'adaptation de leurs comportements, des périodes fiévreuses où la panique s'empare des opérateurs et où, à l'extrême, les prix n'expriment plus aucune information réelle. On est alors face à une défaillance de marché majeure et celui-ci n'est plus à même d'assurer la coordination efficiente des décisions décentralisées. La valeur des actifs concernés, options sur les marchés ou produits physiques, peut ainsi s'écarter durablement de sa valeur définie par les fondamentaux, et se réajuster brusquement.

De telles fluctuations sont sans doute une caractéristique structurelle de l'économie de marché, elles sont coûteuses pour la communauté dans son ensemble. Lorsqu'elles affectent le prix des produits vivriers, elles sont particulièrement néfastes pour les populations les plus pauvres. La stabilité des prix alimentaires est généralement au cœur du contrat social entre les populations et les États, les fluctuations se traduisent alors par une agitation sociale néfaste à l'activité économique et au fonctionnement des marchés.
Les phénomènes cumulatifs en économie sont l'objet de multiples travaux depuis Wicksell au début du XX ${ }^{\mathrm{e}}$ siècle. Ils mettent en évidence les multiples forces « convergentes » et « divergentes » qui font fluctuer le système autour de l'équilibre de la théorie néoclassique et peuvent mener à des déviations cumulatives importantes, suivies de brusques réajustements. Citer tous les travaux concernés ici est impossible, Day [8] en fournit une revue détaillée. Dans les séries économiques obtenues, une dynamique complexe des phénomènes économiques, caractérisée par de fortes fluctuations vient remplacer les simulations stables de l'univers des modèles fondés sur le paradigme walrasien.

Les travaux issus de ces théories conduisent à des modèles mathématiques qui permettent de simuler aussi bien l'économie générale que le seul secteur agricole où le secteur financier. Une différence existe toutefois entre les deux catégories de modèles Dans les approches financières, l'accent est mis sur le seul impact des comportements associés à une certaine volatilité des anticipations, des comportements moutonniers, des phénomènes $d$ 'anticipations auto-réalisatrices. Dans les analyses des prix des matières premières ou de l'économie dans son ensemble, toutes les contraintes susceptibles de s'opposer à l'ajustement (contrainte de liquidité, comportement face au risque, non-négativité des stocks, dépendance des investissements aux résultats passés) jouent un rôle majeur dans la génération de I'instabilité endogène au système. On se trouve alors face à une dynamique économique reposant partiellement sur des processus cumulatifs à la hausse ou à la baisse.

Le modèle de Cobweb (Ezekiel [9]) reproduit de manière très simple le fonctionnement dynamique de la formation des prix dans ce cas (figure 1). L'offre et la demande d'un bien agricole sont respectivement croissante et décroissante avec le prix. Le prix d'équilibre se

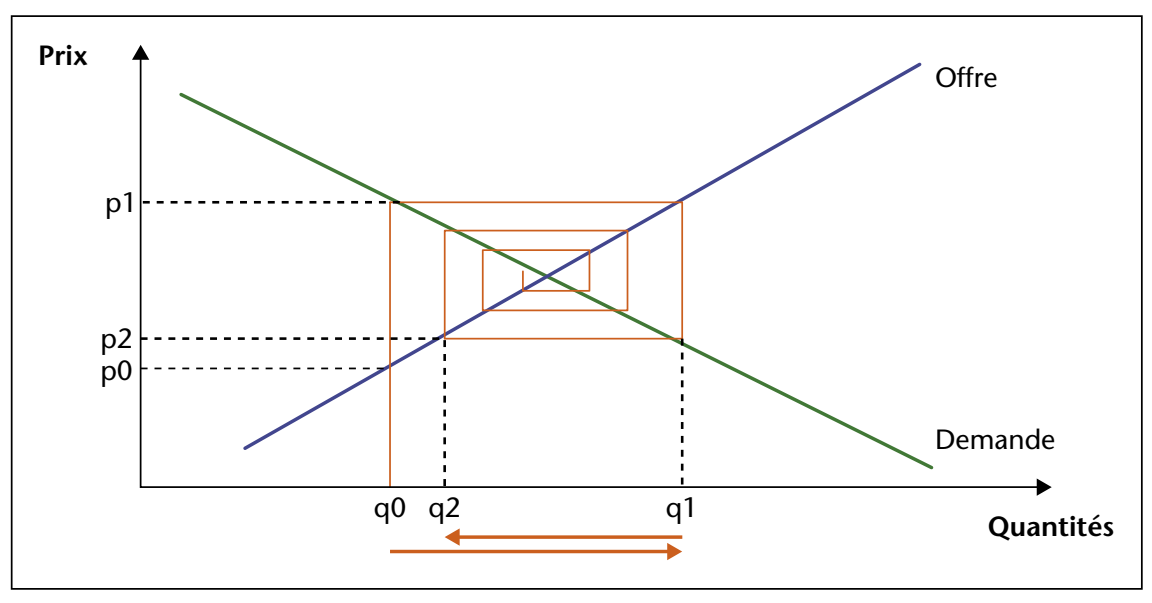

Figure 1. Le Cobweb. 
trouve à l'intersection des deux courbes. Si l'on fait abstraction du temps, cet équilibre est stable. Par contre, si l'on s'intéresse au cheminement vers le point d'équilibre, l'offre et la demande s'ajustant successivement, plusieurs cas doivent être envisagés : lorsque la pente de la courbe de demande est supérieure à celle de la courbe d'offre (en valeur absolue), le marché ne converge pas vers un prix d'équilibre, il s'en éloigne de façon cumulative.

Un tel modèle $n^{\prime}$ est pas complètement satisfaisant car il ne reproduit pas de façon réaliste les comportements des prix réels. Boussard [10] montre qu'en introduisant simplement le risque dans la fonction d'offre d'un Cobweb classique, plus précisément en supposant l'offre est croissante avec le prix mais décroissante avec sa variance [11], les séries de prix obtenues sont tout à fait comparables avec une série de prix agricoles quelconque (figure 2). II montre également que, contrairement à ce que I'on obtient avec des aléas gaussiens, la mise en commun de plusieurs marchés, via le commerce, n'atténue pas les fluctuations, si celles-ci sont générées par le fonctionnement même du marché, mais au contraire les synchronisent ${ }^{1}$.

Une telle conclusion est complètement contraire à celle des "libéraux » qui affirment - en se basant sur la « loi des grands nombres »-que la fusion des marchés trop étroits en un grand marché commun mondial devrait avoir un effet stabilisateur. Ce type d'approche est cependant conforme à ce que Stiglitz [13] nomme « la nouvelle économie du développement » : tout en gardant un cadre général basé sur la théorie néoclassique, on s'affranchit de la référence omniprésente à l'équilibre, pour ouvrir la porte à l'histoire, aux impacts rétroactifs, aux équilibres multiples. Plus inspirée de la biologie que de la mécanique, cette théorie s'intéresse à la dynamique complexe qui semble caractériser les phénomènes socioéconomiques. Cette conception de l'économie dérive directement des conséquences des marchés incomplets, de l'information imparfaite et de la rationalité limitée. Elle est guidée par le constat que les recommandations en matière de politique économique issue du modèle standard ne sont pas systématiquement validées dans la réalité. Ce sera l'objet de la section suivante.

\section{De l'importance économique et politique du choix des modèles}

Il faut bien reconnaître que, en dépit de l'intérêt de ces approches, la plupart des modèles

\footnotetext{
${ }^{1}$ Cf. Boussard et al. [12], p. 21-23 pour plus de détails sur ces questions.
}

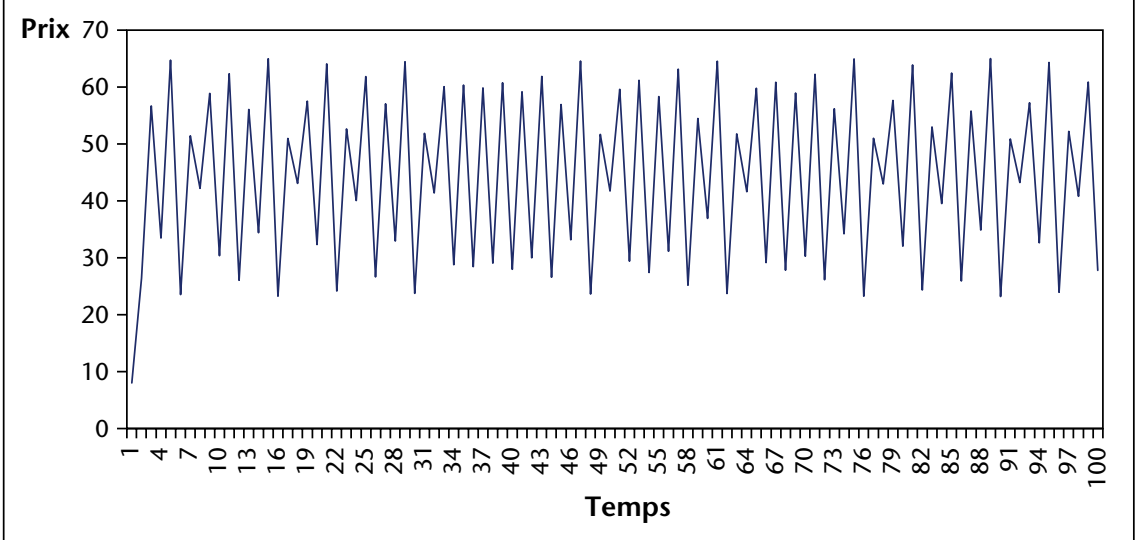

Figure 2. Le Cobweb à risque (demande inélastique). D'après Boussard et al. [12].

utilisés en économie appliquée ne prennent pas en compte cet aspect des choses. Cette section se propose de s'interroger sur les causes et conséquences de cette situation, en revenant tout d'abord à la raison d'être des modèles, puis en examinant leurs prescriptions, dans les négociations internationales comme au niveau des politiques nationales.

À quoi servent les modèles? Les modèles (mathématiques ou non) sont utilisés en sciences comme une représentation cohérente des phénomènes nous permettant de comprendre les mécanismes à l'œuvre, de vérifier que nous les comprenons (validation), et éventuellement d'agir sur eux afin d'en modifier l'évolution. En économie appliquée, ils constituent en principe une aide au « pilotage du système » et à la négociation entre acteurs, ils éclairent la négociation en permettant, s'ils sont chiffrés de synthétiser les impacts des mesures de politique économique par quelques indicateurs clés. Ils permettent ainsi d'évaluer ex ante des mesures alternatives et peuvent constituer des outils précieux dans le contexte de budgets rares de la décision publique.

Les modèles quantitatifs en économie ont été beaucoup développés dans la période récente pour l'analyse des impacts probables de la libéralisation des échanges. À l'origine, ils devaient permettre de justifier l'abandon des politiques de régulation en montrant les effets bénéfiques du marché lorsque celui-ci égalise le prix avec le coût marginal. Si les variantes sont multiples, le moule général est commun et ne considère pas les phénomènes dynamiques décrits ci-dessus. Les milliards de $\$$ de gains annoncés permettaient d'espérer atteindre les objectifs du millénaire et en particulier de diviser par 2 le nombre de pauvres. De fait, le dernier cycle des négociations internationales a été déclaré « cycle du développement ». La déclaration ministérielle de Doha (novembre 2001) indique clairement que les négociations commerciales doivent bénéficier aux pays en développement et tout particulièrement aux pays les moins avancés $(\mathrm{PMA})^{2}$. On s'attendait à ce que ces modèles mettent en évidence la plus grande efficacité du système productif mondial liée à la disparition des distorsions, permettant ainsi un meilleur jeu des avantages comparatifs.

Après deux décennies d'études sur la question, les conclusions peuvent se résumer comme suit :

- Les milliards de $\$$ de gains agrégés associés à la libéralisation totale des échanges iront principalement aux pays développés, l'essentiel de ces gains provient du secteur agricole ${ }^{3}$, ils représentent bien peu en terme de croissance des revenus. En effet si ceux-ci étaient uniformément distribués au sein des pays en développement, selon l'estimation optimiste du modèle Linkage de la Banque mondiale, les gains atteindraient moins de $20 \$$ par an et par tête. Cela fait bien peu au regard de l'étendue du problème de la pauvreté.

- L'essentiel des gains pour les pays en développement ira aux pays émergents (Chine, Inde, Brésil, Argentine). À l'intérieur de ces pays, ce ne sont dans l'ensemble pas les catégories les plus pauvres qui bénéficieront des gains.

- Les pays les plus pauvres d'Afrique subsaharienne sont susceptibles d'être perdants, du fait des impacts négatifs de la croissance des prix agricoles mondiaux sur les ménages acheteurs nets. Ce phénomène touche essentiellement

\footnotetext{
${ }^{2}$ Première page de la déclaration ministérielle, WTO (2001).

${ }^{3}$ Ceci s'explique par le fait que les taxes sur le commerce extérieur sont beaucoup plus importantes pour les produits agricoles, le commerce des biens manufacturés ayant été plus ou moins libéralisé par le passé.
} 
les urbains mais de nombreux ruraux des pays pauvres sont également déficitaires [15].

En dépit de ces résultats peu encourageants en matière de contribution de la libéralisation des échanges à la lutte contre la pauvreté dans les pays les moins avancés, peu d'autres mesures ont été mises en place, dans le cadre des négociations Doha.

Au niveau des politiques nationales, ces modèles prônaient le retrait des États et l'application " uniforme » du consensus de Washington. Deux décennies après la mise en place des premiers plans d'ajustement structurel, on ne peut s'empêcher de constater la faiblesse des progrès enregistrés en matière de lutte contre la pauvreté comme d'amélioration de la sécurité alimentaire. De nombreux experts s'accordent pour souligner le rôle des multiples imperfections de marché, qui se révèlent comme autant d'obstacles à la croissance économique [16, 17]. Alors que le discours dominant des économistes encourageait les pays pauvres à s'insérer dans le commerce mondial, en se spécialisant dans des «niches » comme les fleurs coupées ou des légumes d'exportation et en important les denrées de base, produites à moindre coût ailleurs, avec la flambée des prix des biens alimentaires, on constate les limites de ces prescriptions. Les pays pauvres, moins insérés dans le commerce mondial, s'en sortent mieux comme le montrent les exemples contrastés du Mali et du Sénégal, leur relative indépendance pour l'approvisionnement de leurs populations en denrées de base offre aux États des marges de manœuvre plus importantes pour éviter la contagion sur les marchés locaux.

Si, outre la faiblesse de leur capacité de prévision $^{4}$, les prescriptions issues de nos modèles se révèlent peu efficaces, la question de la validation se pose. Celle-ci soulève, en économie, de nombreux problèmes techniques. De nombreux modèles, étant donné les facilités de calculs ainsi obtenues, sont statiques. Les résultats sont alors à moyen terme et il y a peu de possibilité de les valider : il faudrait que la politique que I'on teste soit mise en place, dans les mêmes conditions que celles utilisées dans la simulation, ce qui est rarement le cas. Par ailleurs, les modèles d'équilibre général utilisent la calibration pour faire correspondre leur situation de départ simulée à l'année de base observée. La validation sur l'année de référence est donc impossible. A priori, la validation des modèles dynamiques devrait poser moins de problèmes et se baser sur la comparaison des résultats de la simulation de référence aux

\footnotetext{
${ }^{4}$ Pour quelques comparaisons édifiantes entre prévisions des modèles économiques et observations voir par exemple Baumel [18].
}

séries réelles disponibles. Cependant, ces modèles n'ont pas l'ambition de représenter les chocs exogènes. Or, lorsque l'on cherche à évaluer si les séries simulées correspondent ou non à la réalité, il y a toujours un « choc extérieur » que le modèle n'a pas l'ambition de représenter et qui peut expliquer la déviation : un choc financier ou climatique par exemple, un progrès technique permettant une croissance de la productivité du travail, etc., qui pourrait expliquer les différences entre les résultats du modèle et la réalité. Comment savoir si le modèle reproduit fidèlement le fonctionnement de l'économie "sans choc » lorsque les seules données que l'on puisse observer sont celles d'une économie fortement touchée par des perturbations externes. ? Ainsi les caractéristiques des modèles les rendent invérifiables au sens de Popper.

À côté de cela, certains experts soulignent les caractéristiques communes des expériences réussies en Asie du Sud-Est en matière de lutte contre la pauvreté et l'insécurité alimentaire comme de croissance économique. Celles-ci sont basées sur une forte intervention de l'État $[19,20]$. Si le détail des politiques menées diffère, deux facteurs communs peuvent être identifiés: (i) une complémentarité entre les mécanismes de marchés et le soutien public dans l'agriculture; (ii) l'importance donnée à la stabilité des prix et au développement de I'infrastructure rurale.

Pour être efficace en matière de lutte contre la pauvreté, l'intervention publique doit permettre la croissance de la productivité du travail, en particulier en zone rurale où se situe l'essentiel des pauvres [14]. Les investissements publics, non seulement dans les infrastructures, comme les routes et les facilités d'irrigation, mais aussi dans le capital humain, avec les services de vulgarisation, ont joué un rôle majeur dans la réussite de plusieurs pays asiatiques, permettant la croissance de la productivité du travail. La stabilisation des prix à un niveau rémunérateur joue sur le même registre, elle permet l'accroissement des investissements privés et protège en même temps les consommateurs les plus pauvres. Un tel contexte a permis à ces économies d'entrer dans un " cercle vertueux »: avec la croissance des revenus ruraux, la demande de bien non agricoles et de services augmente, entraînant la croissance des activités économiques rurales et des opportunités d'emplois avec des conséquences positives sur la lutte contre la pauvreté.

Une telle politique ne serait pas recommandée par nos modèles tels qu'ils sont conçus à I'heure actuelle. Une limite des modèles économiques dominants, même lorsqu'ils sont dynamiques, est qu'ils ne tiennent pas compte de l'incertitude, or c'est l'essence même du temps humain. Les phénomènes économiques sont toujours «explicables après » mais «imprévisibles avant». Cette incertitude explique pourtant une partie des phénomènes économiques. Une autre limite est l'absence de prise en compte des « imperfections de marchés », du capital par exemple, qui supposent la représentation des contraintes de liquidité ${ }^{5}$. Pourtant, le développement, comme la sécurité alimentaire, est le résultat de processus cumulatifs dynamiques et complexes et il semble utile de pouvoir représenter les faits stylisés de ces évolutions. Lorsque les modèles ne prennent pas en compte les défaillances de marché, les politiques économiques sont par définition des contraintes, qui se traduisent par des pertes de bien-être au niveau de la communauté, il semble alors difficile de les utiliser pour évaluer l'intervention.

\section{Tentative à partir d'ID3 : apports et limites}

Faut-il donc considérer que les modèles en général ont échoué à représenter la réalité, et qu'il convient par conséquent d'éviter de s'en servir ? Non, il est sûrement plus intéressant de tenter de modifier les modèles afin de les rendre capables de prendre en compte les phénomènes qui avaient été négligés dans leurs premières versions trop élémentaires. C'est ce qui a été fait depuis plusieurs années avec un modèle mondial dynamique et récursif en équilibre général : ID3 ${ }^{6}$ réalisé au Cirad ${ }^{7}$.

Le modèle fonctionne sur un pas de temps annuel. II est divisé en deux modules : le premier décrit le processus de production physique de biens et services, l'utilisation des facteurs de production dans ce processus, la distribution des revenus associée et la consommation; le second, les opérations financières :

\footnotetext{
${ }^{5}$ Les modèles progressent toutefois dans la représentations de certaines imperfections, ainsi pour le marché du travail, la représentation par des CET du transfert de la main-d'œuvre d'un secteur à l'autre (voir par exemple Hertel et Keeney [21]) ou la prise en compte du chomage [22].

${ }^{6}$ Modèle développé par le Cirad depuis 1999 utilisant la base GTAP 6 (I'année de référence est 2001) qui comprend 8 produits agricoles (blé, autres céréales, élevage bovin, lait, autres productions animales, oléagineux, sucre, autres cultures) et 3 produits agrégés pour représenter le reste de l'économie (manufacture, secteur énergétique, services). Au niveau géographique, deux désagrégations existent : (i) la première en 3 régions (Union européenne, USA, Reste du monde), la seconde en 7 régions (Chine, Inde, Brésil, Union Européenne, USA, PMA, Reste du monde).

${ }^{7}$ Une tentative similaire est depuis peu en cours par le MOMA (Cf : http://www.momagri.org/).
} 
flux de capitaux entre nations, déterminés par l'épargne domestique et le solde commercial et l'allocation des investissements entre les différents secteurs d'activité, en fonction des rentabilités espérées du capital et du risque associé dans les différentes branches de l'économie domestique. II s'agit d'un modèle récursif, c'est-à-dire que chaque année certains paramètres sont mis à jour en fonction des résultats de la période précédente. C'est le cas de la disponibilité en facteurs de production : la disponibilité en capital en début d'année dépend ainsi, outre du capital déjà installé, du niveau d'investissement à la fin de l'année précédente. En outre, dans le modèle en information imparfaite, l'offre agricole en début d'année est déterminée par les choix de production réalisés l'année précédente à partir d'anticipations de prix, révisées chaque année en fonction des prix observés durant les périodes précédentes. Dans la version «standard » les mêmes hypothèses de fonctionnement parfait des marchés de biens que dans les modèles développés par les institutions internationales (Banque mondiale, OCDE etc.) sont retenues. Dans la version avec imperfection de l'information, on suppose que les décisions de production sont prises un an avant la mise sur le marché pour les produits agricoles. Dans cette version, l'offre d'importation, qui s'ajuste immédiatement aux prix, s'ajoute à une offre domestique fixée par les décisions de la période précédente. La production agricole est donc déterminée sur la base de prix anticipés, ce qui génère une demande immédiate en consommation intermédiaire, un niveau d'utilisation des facteurs et un prix pour ceux-ci. Dans ces conditions, tout l'ajustement du côté de l'offre devant se faire essentiellement par les prix, ceux-ci deviennent alors beaucoup plus instables.

De fait, et contrairement à la plupart des modèles « standards » qui génèrent des prix extrêmement stables, on obtient bien ici de fortes fluctuations assez semblables à celles observées dans la réalité. Ce résultat est obtenu en l'absence de tout choc exogène, de toute spéculation, et même de politique en faveur des biocarburants.

Plusieurs scénarios ont été étudiés avec ce modèle. Le scénario de référence est « ref $P A C$ ». II reproduit les réformes européennes récentes (Agenda 2000 et réformes du Luxembourg). Les deux autres scénarii représentent respectivement l'abandon des restitutions aux exportations de la part de l'Union européenne et l'ouverture progressive des marchés conformes aux négociations internationales menées dans le cadre de Doha. Les trois courbes sont pratiquement confondues, que cela soit pour les prix européens (figure 3) ou ceux du reste du monde (figure 4 ). Ce résultat suggère que

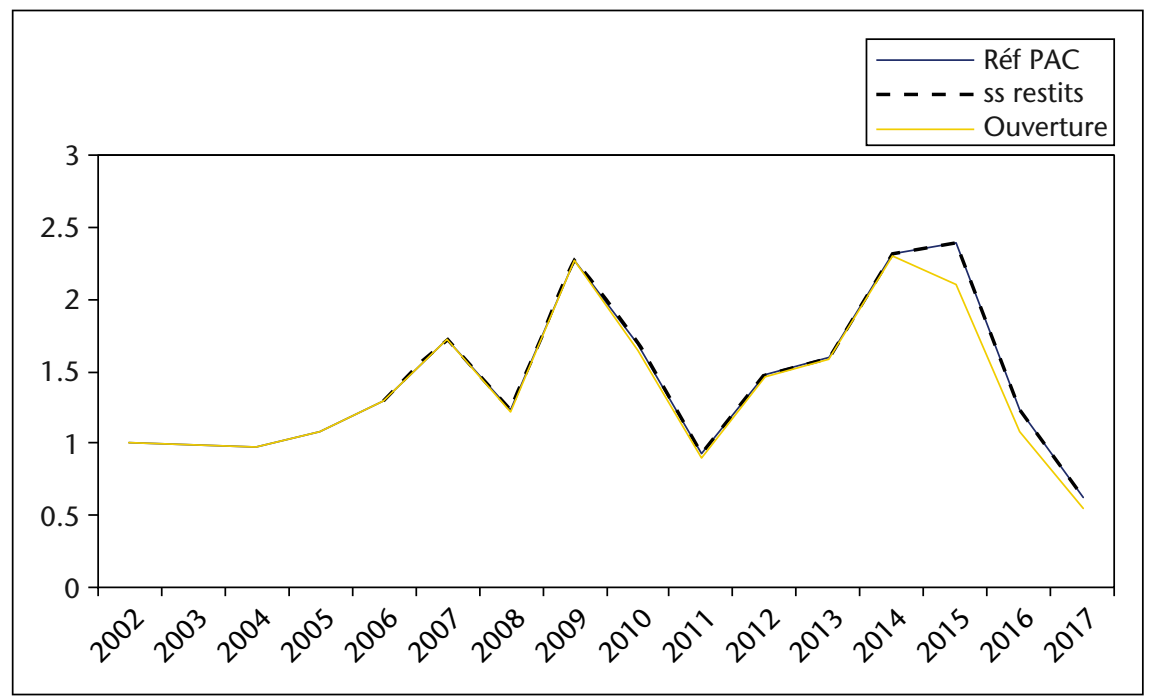

Figure 3. Prix des céréales en UE.

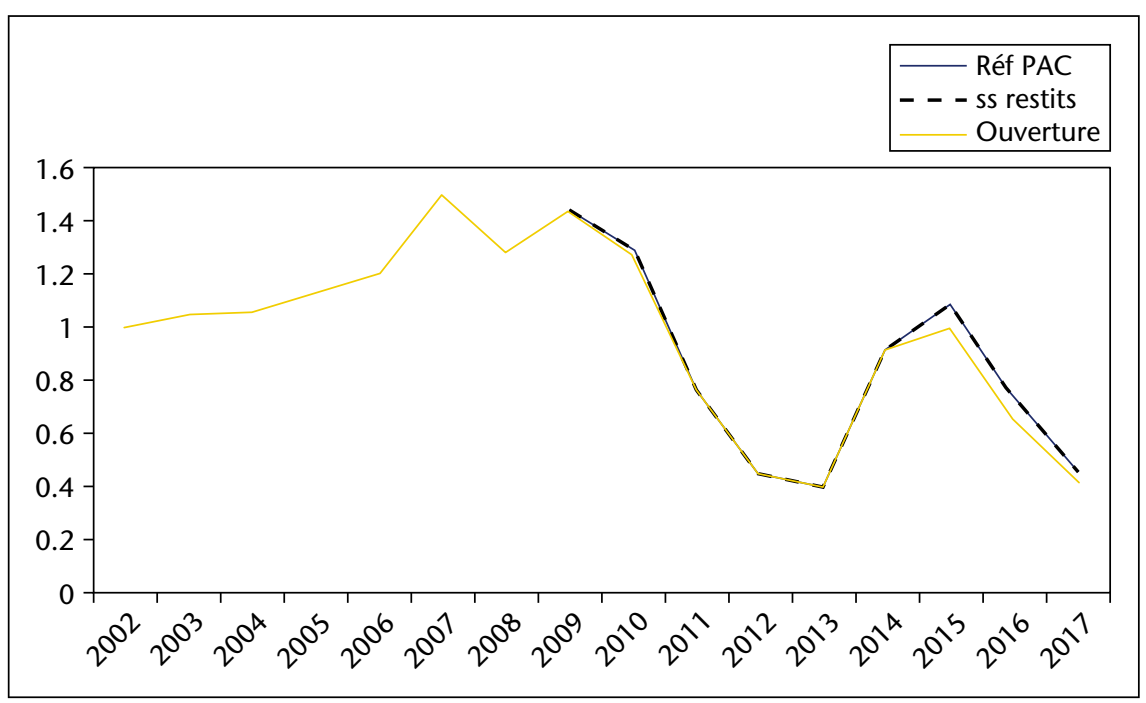

Figure 4. Prix des céréales dans le reste du monde.

l'essentiel des modifications dans l'allure des séries de prix est déjà accompli avec les réformes mises en œuvre en Europe ${ }^{8}$. Mais l'ensemble montre surtout que l'on peut obtenir des séries de prix fluctuantes, simplement en modifiant quelques équations des modèles standards et en introduisant la dérégulation des marchés. Les résultats montrent aussi que l'abandon des subventions aux exportations, largement décriées comme participants de manière significative à l'instabilité des marchés agricoles, ne changent en rien l'allure des courbes.

Les caractéristiques mathématiques de ces modèles font que leurs résultats ne peuvent

\footnotetext{
${ }^{8}$ Pour plus de détails voir Gérard et al. [23] (Une étude réalisée pour le Ministère de l'Agriculture).
}

être assimilés à des prévisions : ni les points de retournement des courbes, ni l'ampleur des fluctuations ne peuvent être déterminées précisément. Cependant, si les comportements représentés sont corrects, l'allure générale des séries nous renseigne sur les dynamiques à l'œuvre. On constate alors que plusieurs pics successifs de prix très élevés sont possibles ainsi qu'une forte chute des prix, au bout de quelques années. Ceci est le résultat des réponses décalées de l'offre, à la fois du fait des délais de production et de l'incertitude. Cela reproduit bien les caractéristiques des matières premières telles qu'elles sont décrites par la littérature, avec une alternance de hausse et de baisse liée aux difficultés d'ajustement de l'offre à la demande, et des effets « ressorts » souvent accentués par les variations de stocks et des 
sur-réactions exacerbées par le jeu des anticipations ${ }^{9}$.

Au niveau des «gains pour le développement ", les résultats obtenus montrent la faiblesse des gains à attendre, en particulier pour les pays les plus pauvres. L'ensemble des experts $s^{\prime}$ accorde d'ailleurs aujourd'hui pour reconnaître que les impacts sur la pauvreté de la libéralisation des échanges agricoles dans les pays les moins avancés seront négligeables au mieux, négatifs dans certains cas où les défaillances de marchés sont trop importantes ${ }^{10}$. II faut donc envisager d'autres modes de redistribution internationale ou d'autres mécanismes à même de promouvoir le développement si on ne veut pas que les objectifs du millénaire ne soient atteints sans les pays les plus pauvres de la planète.

Si le modèle réalisé a bien rempli son objectif initial « montrer la faiblesse des gains de la libéralisation et leur dépendance à de petites variations dans les hypothèses » il reste loin d'être réellement satisfaisant. Le point le plus ennuyeux tient aux difficultés à obtenir des simulations de longues périodes. L'apport de mathématiciens appliqués serait sans doute nécessaire pour améliorer cet aspect.

Le fonctionnement des marchés reste encore trop caricatural, plusieurs pistes d'améliorations existent en la matière : représenter le stockage, différents agents avec des processus $d^{\prime}$ anticipations plus ou moins optimistes pour chaque secteur. Enfin, si l'on considère qu'il est important de représenter avec certains détails les différents types d'agent, car les situations sont variées, il faut aussi disposer de modèles nationaux, pour rester dans des dimensions raisonnables. Puisque l'ensemble des experts s'accordent maintenant sur la faiblesse des gains à attendre de la libéralisation des échanges et que le retrait total des États ne semble pas à même de donner les résultats escomptés en matière de lutte contre la pauvreté, la définition des politiques nationales pourrait bénéficier du développement de ce type de modèle. Compte tenu de l'importance du secteur agricole dans le développement économique et la lutte contre la pauvreté, il semble urgent d'être à même de représenter les processus à l'œuvre sur ces marchés et les principaux déterminants des fluctuations des prix agricoles, ceux-ci apparaissant comme un obstacle essentiel au développement des agricultures locales.

\section{Conclusion}

Cet article analyse la récente flambée des prix sur les marchés de matières premières, comme

\footnotetext{
${ }^{9}$ Calabre [24], page 21.

${ }^{10}$ Voir par exemple, Stiglitz et Charlton [25].
}

le résultat d'un « épisode fiévreux ", caractéristique de ces marchés lorsqu'ils ne sont pas régulés.

Le débat sur les causes de la brusque variation des prix agricoles peut se résumer à une controverse récurrente en économie, aussi bien sur les marchés financiers que sur ceux de matières premières :

- Dans la première conception, les fluctuations sont toujours le résultat d'un signal du marché, elles indiquent une variation dans les fondamentaux, un taux d'endettement dépassant les possibilités de la nation sur les marchés des changes, une sécheresse sur les marchés agricoles, etc. Ces fluctuations ne handicapent pas le fonctionnement des marchés. Elles sont dues à des perturbations extérieures au système et sont réduites par l'élargissement du marché, le changement d'échelle, etc., ce qui permet de les négliger. Dans les modèles, elles sont représentées par un terme aléatoire (gaussien pour les facilités de calculs), ajouté aux fonctions mathématiques.

- Dans la seconde, au contraire, les fluctuations sont le résultat des non-linéarités dans les réponses des agents, des erreurs d'anticipations, des obstacles aux ajustements du marché. Elles sont erratiques et n'amènent aucune information, au contraire elles agissent comme un brouillage des signaux du marché. La représentation de ces phénomènes de "dynamique complexe » est possible dans des modèles économiques, elle est toutefois peu répandue dans les grands modèles considérés par la communauté internationale.

La question peut alors se résumer ainsi : les fluctuations sont-elles exogènes ou endogènes? Négligeables pour la compréhension du système ou au contraire déterminantes ?

Les conséquences économiques de ces deux types de conception des fluctuations sont très différentes. La majorité des modèles supposent qu'elles sont négligeables et les prescriptions qui en découlent sont celles d'une intervention minimale des États dans la vie économique et de la «foi dans les marchés libres » comme politique de développement.

Cependant, il faut bien reconnaître le peu de progrès réalisé, au cours des dernières décennies, dans de la situation des pays à faible revenu, en particulier en Afrique. Les prescriptions issues des modèles dominants en sciences économiques ont donc été peu efficaces, ce qui amène à réfléchir sur leur capacité à décrire correctement les processus et à se pencher sur les possibilités d'améliorations. Le modèle ID3 du Cirad représente une tentative dans cette direction.

\section{RÉFÉRENCES}

1. Mandelbrot L. Formes nouvelles du hasard dans les sciences. Économie Appliquée 1973; 26 : 307-19.

2. Mandelbrot L. Le syndrome de la variance infinie et ses rapports avec la discontinuité des prix. Economie Appliquée 1973 ; 26 : 321-48.

3. Kindleberger CK. Manias, Panics and Crashes. MacMillan, 1996.

4. Merton M. The unanticipated consequences of purposal social action. Am Sociol Rev $1936: 65$.

5. Ravallion M. Markets and Famine. Oxford: Clarendon Press, 1987.

6. Gérard $F$. De la crise financière à la crise alimentaire: I'Indonésie en 1997-1999. Rev TiersMonde 2000 ; XLI (162).

7. FAO/WPF. Crop and food supply assessment mission to Indonesia. Journal of Humanitarian Assistance. Octobre 1998. Site Web: http:// www-jha.sps.cam.ac.uk/b/b127.pdf.

8. Day RH. Complex economic dynamics. Vol 2. The MIT Press, 1999.

9. Ezekiel M. The Cobweb Theorem. Quaterl] Econom $1938 ; 53: 225-80$.

10. Boussard JM. When risk generates chaos. J Econom Behav and Org $1996 ; 29$ : 433-6.

11. Markowitz HM. Portfolio analysis: Efficient diversification of investments. Yale : Yale university press, 1970.

12. Boussard JM, Gerard F, Piketty MG. Libéraliser I'Agriculture Mondiale? Théories, modèles et réalités. CIRAD, 2005.

13. Stiglitz JE. Knowledge for development/Economic Science, Economic Policy, and Economic advice, Paper prepared for the annual world Bank Conference on Development Economics 1998, April 20-21, Waschington DC.

14. Boussard JM, Daviron B, Gérard F, Voituriez T. Agricultural development and food security in subsaharan Africa: Building the case for more support. FAO, 2006

15. World Bank. Development Report 2008.

16. Kherallah M, Delgado C, Gabre-Madin E, Minot $\mathrm{N}$, Johnson M. The road half travelled : agricultural market reform in Subsaharan Africa, IFPRI, Food policy report, 2000.

17. Sitglitz JE. Making globalization work. Londres : Norton Company, 2006.

18. Baumel CP. How US grain exports projections from large scale agricultural sector models compare to reality. Mimeo prepared for the Institute for Agriculture and Trade Policy, Mineapolis, 2001.

19. Stiglitz JE. Some lessons from the East Asian Miracle. The world Bank research observer $1996 ; 11: 151-77$.

20. Timmer CP. The macro dimensions of food security: economic growth, equitable distribu- 
tion, and food price stability. Food Policy 2000 ; $25: 283-95$.

21. Hertel T, Winters LA. Poverty Impact of a WTO agreement: Synthesis and Overview. In : Hertel T, Winters LA, eds. Poverty and the WTO: Impacts of the Doha development Agenda. Washington DC : World Bank, 2006.

22. Polaski S. Winners and losers: impact of the Doha round on developing countries. Washington DC :
Canergie Endowment for International Peace, 2006.

23. Gérard F, Piketty MG, Boussard JM. Analyse de I'impact de scénarios de libéralisation des échanges agricoles internationaux à partir $\mathrm{du}$ modèle ID3. Rapport réalisé pour le ministère de l'Agriculture, 91 p., 2008.

24. Calabre $S$. Régimes de régulation et formes d'organisation des marchés de matières pre- mières. In : Calabre $S$, ed. Matières premières : marchés mondiaux, déséquilibre, organisation. Paris : Economica, 1995.

25. Stiglitz JE, Charlton A. Un cycle de négociations commerciales pour le développement? Revue d'Economie du Développement 2005; 2005 (4) : 18-54.

26. Stiglitz JE. Lessons from East Asia. / Policy Modell $1999 ; 21: 311-30$. 